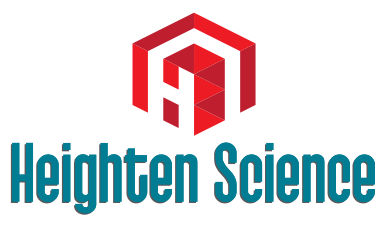

P U B L I C A T I O N S Corporation

\title{
Management of Popliteal Artery aneurysms: Experience in our center
}

\author{
Michiels Thirsa and Vleeschauwer De Philippe* \\ Department of Vascular and Thoracic Surgery, Heilig Hart Ziekenhuis Lier, Belgium
}

*Address for Correspondence: Dr. Vleeschauwer De Philippe, Department of Vascular and Thoracic Surgery, Heilig Hart Ziekenhuis Lier, Belgium, Email: Philippe.De.Vleeschauwer@hhzhlier.be

Submitted: 22 January 2018

Approved: 24 January 2018

Published: 25 January 2018

Copyright: @2018 Thirsa M, et al. This is an open access article distributed under the Creative Commons Attribution License, which permits unrestricted use, distribution, and reproduction in any medium, provided the original work is properly cited

Keywords: Popliteal artery aneurysm; Posterior approach; Open surgical repair

Check for updates

\section{Abstract}

Background: Popliteal artery aneurysms (PAAs) are rare, but the diagnosis should not be missed because of the limb and life threatening complications. The purpose of this study was to reach a consensus about the management of PAA based on our own experience and the available literature.

Materials and Methods: This is a retrospective analysis of all patients who underwent an open surgical PAA repair at our institution from January 2015 to December 2016. Demographic data, risk factors, clinical presentation, aneurysm characteristics, type of repair and results were reviewed. Results include patency and major complications.

Results: Seven patients underwent an open surgical PAA repair (six men). Median age was 72 years. A posterior approach (PA) was chosen four times and a medial approach (MA) was chosen three times. We performed six resections with interposition of a graft and only one ligation with a bypass. Five patients recovered well, did not develop any complication and did not need a second intervention to guarantee patency. These patients underwent a resection of the aneurysm and interposition of a graft (four via a PA and one via a MA). One patient treated by resection and interposition of a Dacron graft via a MA underwent an above-the-knee amputation at postoperative day 14 . This patient had a preoperatively dysfunctional leg since several months with no patent outflow vessels. Our patient treated by ligation and bypass via a MA, developed an acute ischemia four months postoperatively because of an extreme flexion of the knee during several hours while watching TV. After unsuccessful trombolysis, he underwent a femorotibial bypass and a partial forefoot amputation. No longterm results are yet available.

Conclusions: In our opinion, open surgical repair of PAAs by resection of the aneurysm and interposition of a venous graft has the best results. Depending on the relation to the knee joint and thus the accessibility of the aneurysm, a posterior approach is preferred. We are not convinced of endovascular techniques in the treatment of popliteal artery aneurysms.

\section{Introduction}

Popliteal artery aneurysms (PAAs) are the most common of all peripheral artery aneurysms. They can be asymptomatic, and discovered by coincidence, or symptomatic with acute thrombosis or distal embolization. The goal of treatment is limb salvage and to prevent life threatening complications by bypassing or excluding the aneurysm. There are no clear guidelines neither about the time of intervention nor the type of surgery. In the past, open surgery has been the golden standard, but recently endovascular treatment has gained popularity. Because of the lack of large multicenter prospective studies, management remains controversial.

The aim of our study is to reach a consensus about the management of PAAs based on our own experience and the available literature.

\section{Materials and Methods}

Starting January 2015, all patients who underwent surgery for a PAA in our centre were selected for our study using a hypothetico-deductive model. This way we could see if we could falsefy or corroborate our hypothesis that a posterior approach with the interposition technique has the best outcome in the treatment of PAAs. Over a period 
of 24 months, until December 2016, a total of 7 patients met the requirements using a restrictive convenience sample methodology, more specific by judgement sample. All patients were treated by open surgery. Observable data from all the interventions were retrospectively collected by dr. Th. Michiels, resident in General Surgery, including demographic data, risk factors, clinical presentation, aneurysm characteristics, type of repair, results and major complications. In order to determine the characteristics of the aneurysm and the distal outflow, all patients underwent duplex examination and computed tomography angiography (CTA). Indications for surgical repair of PAAs in our centre are: an asymptomatic PAA with a diameter exceeding two $\mathrm{cm}$ or a symptomatic PAA of any size. Asymptomatic patients with a completely thrombosed PAA were not repaired. The characteristics of the aneurysm and the relation of the PAA to the knee joint determined the type of surgical approach. The type of graft was determined by the presence of an appropriate venous graft.

\section{Results}

Demographic data are listed in table 1 . There were six men and one woman. At the time of repair, the median age was 72 (56-90) years. Four patients had an abdominal aortic aneurysm of which three underwent aortic aneurysmectomy. Three patients had a contralateral PAA, but no patient was treated bilaterally. In our female patient, the probable etiology of the PAA was a popliteal entrapment syndrome.

Table 2 describes the aneurysmal characteristics. Two patients were asymptomatic at the time of diagnosis. Among the symptomatic patients, two had calf claudication, one had only a painful popliteal mass, another had a popliteal mass and a drop foot since several weeks and the last patient had a dysfunctional painful leg with a giant popliteal mass since several months. There were no patients with acute ischemia. Three PAAs were fusiform and four were saccular. The mean maximal diameter of the PAA was 4.5 (1.5-12.0) $\mathrm{cm}$ and the mean maximal length was $7.8(2.2-15.0) \mathrm{cm}$. In only three patients, distal runoff status was normal (three patent outflow vessels).

\begin{tabular}{|c|c|}
\hline \multicolumn{2}{|l|}{ Table 1: Demographic data. } \\
\hline Male/female & $6 / 1$ \\
\hline Mean age (years) & $72(56-90)$ years \\
\hline Smoking & $6 / 7$ \\
\hline Hypercholesterolemia & $5 / 7$ \\
\hline Arterial hypertension & $4 / 7$ \\
\hline Known aneurysmal disease & $4 / 7$ \\
\hline Diabetes & $0 / 7$ \\
\hline Bilateral PAA & $3 / 7$ \\
\hline
\end{tabular}

\begin{tabular}{|c|c|}
\hline Table 2: Aneurysmal characteristics. \\
\hline Asymptomatic popliteal artery aneurysm \\
\hline Symptomatic popliteal artery aneurysm \\
Calf claudication & $2 / 7$ \\
\hline Painful popliteal mass & $2 / 7$ \\
Compression symptoms & $3 / 5$ \\
Acute ischemia & $2 / 5$ \\
\hline Aneurysmal configuration & $0 / 5$ \\
\hline Fusiform & $3 / 7$ \\
\hline Saccular & $4 / 7$ \\
\hline Operated side & $1 / 7$ \\
\hline Right & $6 / 7$ \\
\hline Left & \\
\hline Size & $4.5(1.5-12.0) \mathrm{cm}$ \\
\hline Mean maximal diameter (cm) & $7.8(2.2-15.0) \mathrm{cm}$ \\
\hline Mean maximal length (cm) & \\
\hline Distal runoff status & $2 / 7$ \\
\hline 2 patent outflow vessels & $3 / 7$ \\
\hline 1 patent outflow vessels & $1 / 7(2$ distally occluded) \\
\hline no patent outflow vessels & $1 / 7$ (stenosed PTA) \\
\hline
\end{tabular}


All patients underwent an open surgical procedure, of which four times via a posterior approach (PA) (Table 3). In all cases of PA, we performed a resection of the aneurysm and interposition of a graft. The medial approach (MA) was preferred in three cases. Using a MA, we performed two resections of the aneurysm and interposition of a graft. In one case, the aneurysm was ligated and bypassed. We used three saphenous vein grafts, one deep venous graft and three Dacron grafts. A Dacron graft was only used in MA.

Outcomes include patency and major complications during the time of our study period. Five patients recovered well, did not develop any complication and did not need a second intervention to guarantee patency. These patients underwent a resection of the aneurysm and interposition of a graft (four via a PA and one via a MA). One patient treated by resection and interposition of a Dacron graft via a MA underwent an above-the-knee amputation at postoperative day 14. This patient had a preoperative dysfunctional leg since several months with no patent outflow vessels. Our patient treated by ligation and bypass via a MA developed an acute ischemia at four months postoperative because of an extreme flexion of the knee during several hours while watching TV. After unsuccessful trombolysis, he underwent a femorotibial bypass and a partial forefoot amputation. No long-term results of our study patients are yet available (Figure 1).

\section{Discussion}

\section{Background}

An aneurysm is defined as an abnormal permanent dilatation of an artery with at least an increase of $50 \%$ in diameter relative to the normal size of the artery. The size of the popliteal artery in an adult varies between 0.7 and $1.1 \mathrm{~cm}$. The mean growth rate of a PAA is related to the size. For PAAs less than $20 \mathrm{~mm}$ in diameter, the mean growth rate is $1.5 \mathrm{~mm} /$ year, at sizes 20 to $30 \mathrm{~mm} 3.0 \mathrm{~mm} /$ year and at sizes greater than $30 \mathrm{~mm} 3.7 \mathrm{~mm} /$ year $[1,2]$. PAAs are uncommon in the general population with a wide variation of incidence. Nevertheless, the popliteal artery is the second most frequent location of arterial aneurysms after the abdominal aorta and accounting for $70 \%$ of all

\begin{tabular}{|c|c|}
\hline Table 3: characteristics of the surgical intervention. \\
\hline Posterior approach & $4 / 7$ \\
\hline Resection with interposition & $4 / 4$ \\
Ligation with bypass & $0 / 4$ \\
\hline Medial approach & $3 / 7$ \\
\hline Resection with interposition & $2 / 3$ \\
\hline Ligation with bypass & $1 / 3$ \\
\hline Graft type & \\
\hline Saphenous vein & $3 / 7$ \\
\hline Deep vein & $1 / 7$ \\
\hline Dacron graft (all via a MA) & $3 / 7$ \\
\hline
\end{tabular}

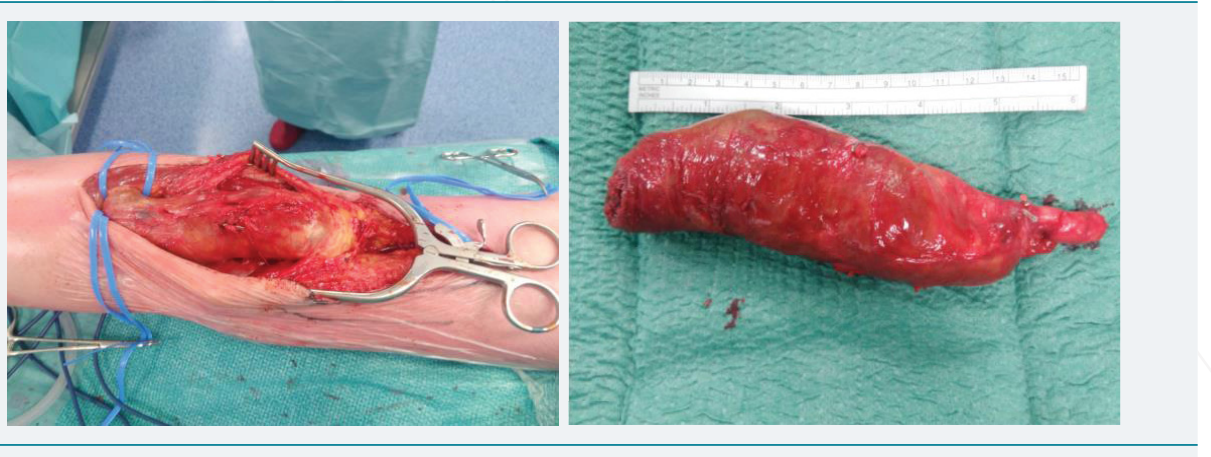

Figure 1: This patient had a painful pulsatile mass in the knee pit with a drop foot. He was diagnosed with a fusiform aneurysm of $4.3 \times 15.0 \mathrm{~cm}$. We performed an open resection via posterior approach with interposition of a deep venous graft. Photos by dr. De Vleeschauwer. 
peripheral aneurysms. Males are 20 times more affected than females and the mean age at presentation is 65 years. The prevalence increases with age. $50 \%$ of the patients have a PAA in the contralateral leg and $33 \%$ has an abdominal aortic aneurysm (AAA) [3-5]. This implicates the cost-effectiveness of screening patients with a PAA for AAA. In patients with an AAA, a concomitant PAA is present in only $3 \%$ [6].

Our patient population had a mean age of 72 years, which is older than the mean age reported in other studies. As seen in literature, there was indeed a male predominance and almost half of our patient population ( $42 \%$ ) had a bilateral PAA.

The etiopathogenesis is complex and probably multifactorial, although arteriosclerosis seems to be the main cause in elderly patients. Male gender, smoking and family history are the most important risk factors. In young patients, a PAA is rare and mostly caused by popliteal artery entrapment, trauma, cystic adventitial degeneration or inflammatory and collagenous diseases like Marfan and Behçet. The diagnosis of a PAA is very important because of the high risk of limb dysfunction and limb loss once complications occur. About $45 \%$ of the patients with PAAs are asymptomatic at time of diagnosis. In contrast to our study, the majority of patients (71\%) were symptomatic. Every year, about $14 \%$ of patients with an asymptomatic PAA develop symptoms, being local mass effect or ischemia of the leg $[2,6]$. A PAA can locally cause a painful pulsatile mass in the knee pit, ischiadic nerve dysfunction or deep venous thrombosis due to compression of the adjacent neurovascular structures [2,7].

Ischemic effects include chronic limb ischemia (claudication) on one hand and acute ischemia of the leg on the other hand. Acute ischemia, being a main complication, occurs in about one-third of the symptomatic patients within five years and is usually associated with thrombosis, distal embolization or rarely, rupture [8,9]. To establish the diagnosis of a PAA, clinical examination and imaging techniques are used. A pulsatile mass is not always easily palpable because of its deep position in the popliteal fossa.

Duplex examination is most commonly used for the diagnosis of a PAA. It gives an idea about the patency of the popliteal artery, the dimensions of the PAA and the presence of a thrombus. A CTA or magnetic resonance angiography (MRA) is more precise and is often performed for planning the operative approach. They also provide information about the size of the adjacent arterial segments and the distal runoff status. They are less invasive, less expensive, contain fewer risks and give less discomfort than a conventional arteriography, which was the golden standard in the past [10].

There is a consensus that all symptomatic PAAs, despite the diameter, should be treated because of the highly increasing risk of severe complications once symptoms are present. Unfortunately there are no clear guidelines concerning the treatment of asymptomatic PAAs. There is some consensus that a conservative management of asymptomatic PAAs with a diameter less than $2 \mathrm{~cm}$ is safe because of a low incidence $(0-9 \%)$ of complications [3,11]. This means that in general, and to our opinion, a diameter greater than $2 \mathrm{~cm}$ is an indication for elective surgery in asymptomatic PAAs.

Besides the diameter of the PAA, the presence and size of a thrombus, a poor distal runoff status and a high degree of distortion are risk factors for developing symptoms in patients with an asymptomatic PAA. These factors should favour elective surgical repair of the PAA $[2,12]$. Others suggest that all PAAs, even asymptomatic ones, should be treated because of the low operative risk in the elective setting [13]. An important consideration is that the outcomes of surgery in asymptomatic PAAs are better than in symptomatic PAAs $[11,14,15]$. This is something we can confirm in our patient population: the patients who were most symptomatic, had the worst outcome. Some authors suggest that elective surgery has a better outcome than emergency surgery. There are also studies in which the outcome of PAA repair was comparable in both settings [16]. 
Unfortunately there is a lack of clear guidelines in choosing the surgical technique for PAA repair. The primary goal of treating a PAA is the prevention of thromboembolism and amputation. The secondary goal is to prevent further enlargement of the aneurysmal sac, which can cause compression symptoms on adjacent structures or which can lead to rupture.

There are two main options for operative management: open surgery, which has been the golden standard for many years, and the recently upcoming endovascular treatment. The surgical decisionmaking in a PAA is multifactorial and complex. It depends on factors such as the size and configuration of the PAA, the distal runoff status, the availability of a venous graft and the comorbidities of the patient.

Open surgery involves excluding the aneurysm by creating a bypass or an interposition graft, either with an autologous vein (most commonly the great saphenous vein) or a prosthetic graft when adequate venous material is not available. There are several approaches and techniques, but the posterior approach (PA) and medial approach (MA) to the popliteal space are the most commonly used. The relation of the PAA to the knee joint, the size and the configuration determine the decision between these two techniques. In PAAs above the knee joint, a MA is technically more feasible.

In the bypass technique, the proximal and distal ends of the aneurysm are identified, ligated and a bypass will be created. The aneurysm will be left in situ and excluded from the circulation. A disadvantage of this technique is that after ligation, there is a risk of progressive growth of the aneurysm due to persistence of small side-branches that provide retrograde filling of the aneurysmal sac. Therefore all collaterals should be identified and ligated as well [14].

In our center we prefer a posterior approach and the interposition technique with an autologous vein if possible. In this technique, complete dissection and resection of the aneurysm is performed. An endto-end interposition graft is then created in an anatomical position, which provides good hemodynamics. For this technique, when using an autologous vein, only a short segment needs to be harvested. Preferably the great saphenous vein is used, because the calibre of the small saphenous vein is often inadequate.

When a PA is chosen, the patient is installed in ventral decubital position. When using a vein graft in the PA, an extra incision and turning of the patient during surgery is required. When a MA is preferred, the patient is installed in dorsal decubital position. The incisions are made at the medial side of the leg, above and below the knee joint. Harvesting the great saphenous vein can take place in the same patient position and the vein can be used as an in situ bypass or as a reversed vein bypass. The MA has fewer risks for iatrogenic neurovascular trauma. This approach also provides easier access to the distal arteries.

Cervin et al., performed a large study in Sweden to compare the outcomes of the different surgical techniques used in PAA repair. Table 4 shows the results of this comparison (349 interventions via a MA and 121 via a PA). They do not specify

\begin{tabular}{|c|c|c|c|c|c|c|c|c|c|c|c|c|}
\hline \multirow{2}{*}{$\begin{array}{l}\text { Indication } \\
\text { Surgical approach }(N)\end{array}$} & \multicolumn{2}{|c|}{ All procedures } & \multirow[t]{2}{*}{$p$} & \multicolumn{2}{|c|}{ Acute ischaemia } & \multirow[t]{2}{*}{$p$} & \multicolumn{3}{|c|}{ Elective symptomatic $p$} & \multicolumn{3}{|c|}{ Elective asymptomatic $p$} \\
\hline & Medial 349 & Post. 121 & & Medial 112 & Post. 24 & & Medial 73 & Post. 17 & & Medial 164 & Post. 80 & \\
\hline Prim. patency $(N)$ & $309 / 340$ & $118 / 120$ & .007 & $91 / 105$ & $22 / 23$ & .225 & $66 / 72$ & $17 / 17$ & .2181 & $152 / 163$ & $79 / 80$ & .063 \\
\hline 30 days $(\%)$ & 90.9 & 98.3 & & 86.7 & 95.7 & & 91.7 & 100 & & 93.3 & 98.8 & \\
\hline Sec. patency $(N)$ & $325 / 341$ & $120 / 121$ & .052 & $97 / 105$ & $23 / 24$ & 0.549 & $67 / 72$ & $17 / 17$ & .2631 & $161 / 164$ & $80 / 80$ & .224 \\
\hline 30 days $(\%)$ & 95.3 & 99.2 & & 92.4 & 95.8 & & 93.1 & 100 & & 98.2 & 100 & \\
\hline Amputations $(N)$ & $8 / 345$ & $0 / 121$ & .091 & $5 / 108$ & $0 / 24$ & 0.283 & $3 / 73$ & $0 / 17$ & $.395 c$ & 0 & 0 & - \\
\hline 30 days (\%) & 2.3 & 0 & & 4.6 & 0 & & 4.1 & & & & & \\
\hline Prim. patency $(N)$ & $247 / 296$ & $89 / 101$ & .261 & $71 / 91$ & $18 / 22$ & 0.696 & $48 / 60$ & $12 / 14$ & .6231 & $127 / 144$ & $59 / 65$ & .582 \\
\hline 1 year $(\%)$ & 83.4 & 88.1 & & 78.0 & 81.8 & & 80.0 & 85.7 & & 88.2 & 90.8 & \\
\hline Sec. patency $(N)$ & $267 / 297$ & $95 / 104$ & .668 & 79/91 & $19 / 22$ & 0.956 & $51 / 60$ & $13 / 14$ & .4391 & 137/146 & $63 / 68$ & .743 \\
\hline 1 year $(\%)$ & 89.9 & 91.3 & & 86.8 & 86.4 & & 85.0 & 92.9 & & 93.8 & 92.6 & \\
\hline Amputations $(N)$ & $17 / 308$ & $0 / 109$ & .012 & $8 / 94$ & $0 / 22$ & 0.156 & $7 / 65$ & $0 / 16$ & .1702 & $2 / 149$ & $0 / 71$ & .327 \\
\hline 1 year (\%) & 5.5 & 0 & & 8.5 & 0 & & 10.8 & 0 & & 1.3 & 0 & \\
\hline
\end{tabular}


whether a bypass or an interposition graft is used. A distinction is made between indication of the procedure: elective surgery in symptomatic patients, elective surgery in asymptomatic patients or surgery in case of acute ischemia. There was no significant difference in amputation and patency rates after 30 days and 1 year between the MA and PA in the three sub-groups. Growth of the aneurysm is a late complication of the MA, which develops many years after the primary operation. This phenomenon could not be expected to occur within the 1year follow-up period of this study. They also compared outcomes after open repair depending on graft material. The use of a vein graft resulted in a better patency in all sub-groups but the benefit was greatest in patients with acute ischemia [17].

Also Mazzaccaro et al., conducted a retrospective study comparing PA and MA in a total of 77 PAAs. In all the PA repairs (43 patients), a resection of the aneurysm and an interposition of a graft was performed. Using MA repair (34 patients), 22 resections of the aneurysm and interposition grafts, and 12 bypasses were performed. A prosthetic graft was used in a total of 54 cases. The PA and MA patients differed significantly in age (the MA group being older) and smoking history (more frequent in the PA group). They found that both approaches had satisfactory results concerning 5-year primary and secondary patency rates and limb salvage. The differences between both groups were statistically not significant. There was a similar postoperative nerve and wound complication ratio in both groups. Only the in-hospital stay after PA was significantly lower $[18,19]$.

Ravn et al., also investigated the importance of the surgical technique of PAA repair on long-term outcomes. When comparing all patients in the PA- and MA-group, the growth of the PAA was greater in the MA-group than in the PA-group because of the difficult exposure of the side-branches and thus lack of ligating them. Age, emergency procedure and prosthetic grafts were independently associated with a higher longterm amputation rate (Table 5).

Since 1994, some surgeons choose to treat PAAs by endovascular techniques (ET). At present, large studies reporting long-term outcomes are lacking, but mandatory. In this technique, the popliteal artery is approached by punction of the common femoral artery and a stent graft is positioned to exclude the PAA. It offers the advantage of

\begin{tabular}{lccr}
\hline & Veingmaft & Prosthetic graft & $\mathrm{P}^{*}$ \\
\hline 30 days & & & \\
PAG & $92 \%$ & $86 \%$ & .664 \\
MAG & $94 \%$ & $87 \%$ & .016 \\
$P^{*}$ & .498 & 1.0 & \\
One year & & & \\
PAG & $85 \%$ & $81 \%$ & .719 \\
MAG & $90 \%$ & $72 \%$ & $<.001$ \\
$P^{*}$ & .374 & .584 & \\
At re-examination & & & \\
PAG & $90 \%$ & $57 \%$ & .101 \\
MAG & $84 \%$ & $69 \%$ & .098 \\
$P^{*}$ & .743 & .661 & \\
\hline
\end{tabular}

$P A G$, Posterior approach group; $M A G$, medial approach group.

*Refers to Fischer exact test, comparing graft type within the posterior or medial approach groups.

${ }^{t}$ Refers to Fischer exact test, comparing the posterior or medial approach groups, within the subgroups with same graft type.

Table 5: Primary patency at different time points depending on the surgical approach and the type of graft used (Ravn et al.). 
being less invasive and having less wound complications and blood loss. This technique provides a quicker recovery and a shorter operative time. General anaesthesia can also be avoided. High-risk surgical patients with severe comorbidities can benefit from this type of repair. The ET is generally contraindicated in young fit-for-surgery patients, patients with poor distal runoff status, compression symptoms and patients with a contraindication to antiplatelet, anticoagulant, or thrombolytic therapy. The main problem of the ET is the required flexibility of the stent graft because of the close relation with the knee joint. Secondly, the proximal and distal neck of the aneurysm need to provide a secure site for fixation of the stent graft. Thirdly, embolisation of significant collateral vessels needs to prevent enlargement of the aneurysmal sac [17,20-23].

Endograft thrombosis is the major complication in the ET. This risk can be decreased by careful patient selection based on strict anatomic criteria and by improvement in stentgraft design and flexibility. Also migration, stenosis at the border of the stent, continuous sac enlargement and breakage of the stent material are possible complications $[17,20-23]$.

Several, mostly retrospective, studies have investigated the advantages of the ET. Antonello et al. investigated in a prospective randomized study the risks and advantages of using a Hemobahn graft (a self-expanding nitinol stent internally covered by an ultra-thin PTFE-graft) compared with open repair. The preferred open technique was ligation of the aneurysm and bypass through a MA. Both groups contained 15 patients and all were asymptomatic. There was no statistical difference in primary and secondary patency rates at 1 year between the two groups. This may be the result of patient selection and the postoperative therapy. Only patients with optimal anatomic characteristics for ET and with a good runoff status were enrolled. The mean hospital stay and length of surgical procedure were significantly longer in open surgery, which could make ET more appropriate in high- risk patients [20].

Tielliu et al., also performed a prospective cohort study to evaluate suitability of the ET. 57 PAAs were treated endovascularly. 12 were symptomatic, and five of them had an acute ischemia, which were treated with trombolytic therapy before stenting. During a mean follow-up of 24 months, 12 stent grafts occluded. None of these patients required amputation or bypass surgery. Primary and secondary patency rates were $80 \%$ and $90 \%$ at 1 year and $77 \%$ and $87 \%$ at 2 years follow-up. They conclude that patency rates in ET are slightly inferior to the open repair in the literature. Postoperative treatment with clopidogrel proved to be the only significant predictor for success [21].

Pulli et al. conducted a retrospective multicenter study. 178 open surgical interventions and 134 endovascular exclusions for PAA were performed. In the open group, there were 13 open resections of the aneurysm with interposition of a graft, 73 aneurysms were opened combined with inlay placement of a graft and in the other 92 cases ligation with bypass creation was carried out. They used a MA in 139 cases and a PA in 39 cases. One important remark is that both groups were not homogenous. The mean age of the endovascular group was higher. The patients in the open group were more symptomatic, had more frequently acute limb ischemia and an overall worse distal runoff status. Since the indication to open repair and ET significantly differed, they did not want to perform a direct comparison between the two techniques. This study concludes that both methods are feasible and safe, but that they are used in different patients with regard to clinical and anatomical characteristics [22]. Cervin et al., demonstrated that patency in ET is inferior to the open repair, as well for asymptomatic as for symptomatic patients, especially after treatment for acute ischaemia. Also the amputation risk tends to be higher in ET despite similar pre-operative characteristics [17].

Von Stumm et al. studied 652 cases of PAA. This currently is the largest meta-analysis in the comparison of ET with open surgery. He included five retrospective studies. ET showed mid-term results comparable to open surgery, but the 30-day reintervention and thrombosis rate in ET were higher than in open surgery. An important remark 
is that patients of the ET group were significantly older and less symptomatic than surgically treated patients, which is in line with the previous literature. The included studies were all retrospective, rather small and did not have an identical study protocol. The follow-up periods were short and lost to follow-up rates were high. Von Stumm concludes that ET appears to be a feasible and safe alternative in selected patients, although the quality of evidence is low [23].

We have several remarks on this topic. First of all, most studies on open surgery in PAAs compare the results of a MA with a PA. Studies comparing ligation of the aneurysm with bypass and resection of the aneurysm with interposition of a graft, independently of the approach, are bearly available in the literature. Secondly, we found out that a high quality comparison between ET and open surgery is currently not available because of the heterogeneity of patients included in both groups. There is also a difference between experience in treatment of PAAs in endovascular highly specialized centers and classical centers. Therefore, in our opinion, ET cannot yet be considered as a routine treatment of PAA. Prospective large multicenter randomized controlled trials with long-term follow-up are necessary, although difficult to accomplish because of the low prevalence of PAAs.

\section{Conclusion}

In the past, the most common treatment of PAA was resection of the PAA with interposition of an autologous vein using a PA to the popliteal fossa. Based on our own experience in our centre this technique has the best results. Although many other techniques and approaches have developed over the years, we are convinced that this treatment remains the golden standard for the treatment of PAAs.

\section{References}

1. Pittathankal A, Dattani R, Magee T, Galland R. Expansion Rates of Asymptomatic Popliteal Artery Aneurysms. Eur J Vasc Endovasc Surg. 2004; 27: 382-384. Ref.: https://goo.gl/DnDAvb

2. Galland R. Popliteal Aneurysms: From John Hunter to the 21st Century. Ann R Coll Surg Engl. 2007; 89: 466-471. Ref.: https://goo.gl/4vdCYe

3. Szilagyi DE, Schwartz RL, Reddy DJ. Popliteal Arterial Aneurysms. Arch Surg. 1981; 116: 724. Ref.: https://goo.gl/oQkzXi

4. Anton G, Hertzer N, Beven E, O'Hara P, Krajewski L. Surgical management of popliteal aneurysms. J Vasc Surg. 1986; 3: 125-134. Ref.: https://goo.gl/XTdjYL

5. Carpenter J, Barker C, Roberts B, Berkowitz H, Lusk E, et al. Popliteal artery aneurysms: Current management and outcome. J Vasc Surg. 1994; 19: 65-73. Ref.: https://goo.gl/oVDSQq

6. Michaels J, Galland R. Management of asymptomatic popliteal aneurysms: The use of a markov decision tree to determine the criteria for a conservative approach. Eur J Vasc Surg. 1993; 7: 136143. Ref.: https://goo.gl/tJfUUP

7. Claridge $M$, Hobbs S, Quick C, Adam D, Bradbury A, et al. Screening for popliteal aneurysms should not be a routine part of a community-based aneurysm screening program. Vasc Health Risk Manag. 2006; 2: 189-191. Ref.: https://goo.gl/yjBif8

8. Bowyer R, Cawthorn S, Walker W, Giddings A. Conservative management of asymptomatic popliteal aneurysm. Bri J Surg. 1990; 77: 1132-1135. Ref.: https://goo.gl/4mRzq2

9. Neiman H, Yao J, Silver T. Gray-Scale Ultrasound Diagnosis of Peripheral Arterial Aneurysms. Radiology. 1979; 130: 413-416. Ref.: https://goo.gl/xzm3k1

10. Rizzo R, Flinn W, Yao J, McCarthy W, Vogelzang R, et al. Computed tomography for evaluation of arterial disease in the popliteal fossa. J Vasc Surg. 1990; 11: 112-119. Ref.: https://goo.gl/1XvQRL

11. Schellack J. Nonoperative Management of Selected Popliteal Aneurysms. Arch Surg. 1987; 122 372-375. Ref.: https://goo.gl/qS9HD2

12. Lowell R, Gloviczki P, Hallett J, Naessens J, Maus T, et al. Popliteal Artery Aneurysms: The Risk of Nonoperative Management. Ann Vasc Surg. 1994; 8: 14-23. Ref.: https://goo.gl/N2moXQ 
13. Shortell C, DeWeese J, Ouriel K, Green R. Popliteal artery aneurysms: A 25year surgical experience. J Vasc Surg. 1991; 14: 771-779. Ref.: https://goo.gl/o7XTTk

14. Mahmood A, Salaman R, Sintler M, Smith S, Simms M, et al. Surgery of popliteal artery aneurysms: A 12-year experience. J Vasc Surg. 2003; 37: 586-593. Ref.: https://goo.gl/N5dGYU

15. Dawson I, Sie R, van Bockel J. Atherosclerotic popliteal aneurysm. Bri J Surg. 1997; 84: 293-299. Ref.: https://goo.gl/CQqBFo

16. Aulivola B, Hamdan A, Hile C, Sheahan M, Skillman J, et al. Popliteal artery aneurysms: a comparison of outcomes in elective versus emergent repair. J Vasc Surg. 2004; 39:1171-1177. Ref.: https://goo.gl/4WhPMi

17. Cervin A, Tjärnström J, Ravn H, Acosta $S$, Hultgren R, et al. Treatment of Popliteal Aneurysm by Open and Endovascular Surgery: A Contemporary Study of 592 Procedures in Sweden. Eur J Vasc Endovasc Surg. 2015; 50: 342-350. Ref.: https://goo.gl/Jz6ujV

18. Mazzaccaro D, Carmo M, Dallatana R, Settembrini A, Barbetta I, et al. Comparison of posterior and medial approaches for popliteal artery aneurysms. J Vasc Surg. 2015; 62: 1512-1520. Ref.: https://goo.gl/aDVS5F

19. Ravn $\mathrm{H}$, Wanhainen $A$, Björck $M$. Surgical technique and long-term results after popliteal artery aneurysm repair: Results from 717 legs. J Vasc Surg. 2007; 46: 236-243. Ref.: https://goo.gl/JrnAwt

20. Antonello M, Frigatti P, Battocchio P. Open Repair versus Endovascular Treatment for Asymptomatic Popliteal Artery Aneurysm: Results of a Prospective Randomized Study. J Vasc Surg. 2005; 42: 185193. Ref.: https://goo.gl/Qb1tEc

21. Tielliu I, Verhoeven E, Zeebregts C. Endovascular Treatment of Popliteal Artery Aneurysms: Results of a Prospective Cohort Study. J Vasc Surg. 2005; 41: 561-566. Ref.: https://goo.gl/t1HREB

22. Pulli R, Dorigo W, Castelli P, Dorrucci V, Ferilli F, et al. A Multicentric Experience with Open Surgical Repair and Endovascular Exclusion of Popliteal Artery Aneurysms. Eur J Vasc Endovasc Surg. 2013; 45: 357-363. Ref.: https://goo.gl/xjJ3bJ

23. Von Stumm M, Teufelsbauer H, Reichenspurner H, Debus E. Two Decades of Endovascular Repair of Popliteal Artery Aneurysm-A Meta-analysis. Eur J Vasc Endovasc Surg. 2015; 50: 351-359. Ref.: https://goo.gl/KGMnNk 